Mitteilungen der Österreichischen Geographischen Gesellschaft,

155. Jg. (Jahresband), Wien 2013, S. 125-147

\title{
Anmerkungen zur Variabilität von Migrationssystemen (mit Erfahrungen aus Lettland und Albanien). Transnationalismus Oder TransRegionalismus? ${ }^{1)}$
}

\author{
Daniel Göler, Bamberg, und Zaiga KRIŠJĀNE, Riga [Rīga]* \\ mit 3 Abb. und 2 Tab. im Text
}

INHALT

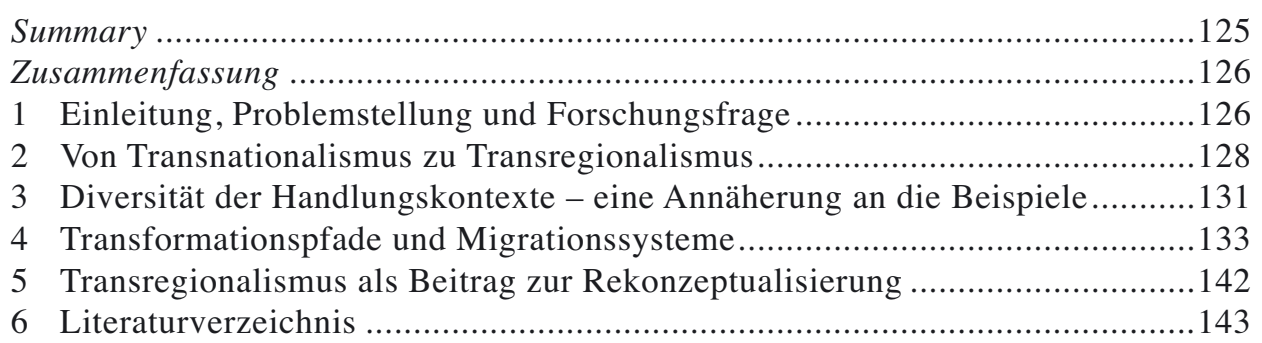

\section{Summary}

On the variability of migration systems (with experiences from Latvia and Albania). Transnationalism or transregionalism?

The global financial and economic crisis as well as post-socialist transition meant a similar general framework for socio-economic development. The outcome of such externally caused incidents show in similar contexts quite different reactions. The study focuses on migration systems which are marked by a wide-spread variability and diversity. Our case studies on Latvia and Albania show different preconditions in their status regarding EU-membership as well as various types of predominant

\footnotetext{
1) Die Studie entstand im Rahmen eines Humboldt-Stipendiums an der Professur für Geographische Migrations- und Transformationsforschung an der Otto-Friedrich-Universität Bamberg. Die Autoren sind der Alexander-von-Humboldt-Stiftung in tiefstem Dank verbunden.

* Prof. Dr. Daniel GöLER, Institut für Geographie, Universität Bamberg, Geographische Migrations- und Transformationsforschung, Am Kranen 12, D-96045 Bamberg, Deutschland; E-Mail: daniel.goeler@ uni-bamberg.de; http://www.uni-bamberg.de/geographie/migtrans/; Prof. Dr. Zaiga KRIŠJĀNE, Institut für Geographie, Universität von Lettland, Alberta iela 10, Rīga, LV-1010, Lettland; E-Mail: zkrisjan@ lanet.lv; http://www.geo.lu.lv/geografijasnodala/
} 
population mobility: While Latvia is characterised by stable permanent emigration, Albania emphasises mass-outmigration with higher potentials of return. Empirical findings indicate several key elements which are not sufficiently analysed in previous research on migration and mobility. We suggest to broadening the concept of transnationalism by a new transregional perspective in order to strengthen the spatial issue in migration studies.

\section{Zusammenfassung}

Die globale Finanz- und Wirtschaftskrise definiert, ebenso wie es die post-sozialistischen Transformationsereignisse tun, scheinbar einen gleichen Handlungsrahmen für alle. Trotz, ihrer ähnlichen Wirkmacht führen solche extern verursachten Ereignisse selbst in vergleichbaren Kontexten aber zu sehr unterschiedlichen Reaktionen. Mit Blick auf Migrationen im Rahmen post-sozialistischer Transformationen wird deshalb, bei aller Diversität der Objekte, der Frage der Variabilität von Migrationssystemen nachgegangen. Die Beispiele Lettland und Albanien sind sowohl hinsichtlich der Rahmenbedingungen (EU-Mitglied versus Nicht-EU-Mitglied) und der jeweiligen Migrationsformen (stabile Emigration mit dauerhaften Zügen versus Massenemigration mit hohem Rückkehrpotenzial) sehr unterschiedlich. In den empirischen Befunden kristallisieren sich differenzierende Elemente heraus, welche in migrationstheoretischen und raumbezogenen Debatten wenig berücksichtigt werden. Mit der vorgeschlagenen Erweiterung des Transnationalismus-Konzeptes um eine transregionale Komponente bereichern wir die Migrationsforschung mit neuen Argumenten.

\section{Einleitung, Problemstellung und Forschungsfrage}

Globalisierung und Transformation brachten um die Jahrtausendwende mannigfaltige politische, soziale und ökonomische Veränderungen sowie neue Formen von Mobilität und Migration mit sich. So hat sich die Arbeitsmigration mit den EU-Erweiterungen und zuletzt durch die weltweite Finanz- und Wirtschaftskrise intensiviert. Vor diesem Hintergrund entfalten und verändern sich die Interaktionen zwischen den Herkunftsund Zielregionen von Migranten. Sie stehen im Mittelpunkt der folgenden Argumentation. Ausgangspunkt ist die Feststellung, dass die genannten Globalereignisse in unterschiedlichen räumlichen und sozialen Kontexten nicht zwingend zu gleichen Reaktionen führen oder geführt haben. So fielen die Transformationspfade nach dem Ende der - ihrerseits bereits mit zahlreichen individuellen Spielarten ausgestalteten - sozialistischen Planwirtschaft im östlichen Europa höchst unterschiedlich aus (Smith \& Timár 2010; Pickles 2010; Pickles \& Smith 1998). Leidlich erfolgreichen Transformationen (z.B. in Tschechien, Polen, den baltischen Republiken) stehen unterbrochene (Albanien), verzögerte (Serbien) oder bislang gescheiterte Pfade (Bosnien und Herzegowina) gegenüber. Noch viel mehr gilt das, was Migration betrifft, für die Implikationen der jüngeren Krise (FIndLAY et al. 2010; Fielding 2010; Hillmann et 
al. 2012). Deren räumlich differenzierender Charakter ist bislang wenig beforscht. Insofern erscheint es reizvoll, in diesem Zusammenhang zwei sehr unterschiedliche Migrationssysteme (namentlich Albanien und Lettland) zu analysieren.

Für entsprechende Studien im Rahmen von Migration und Transformation ergibt sich eine Reihe relevanter und über Themen wie Integration und Migrationsnetzwerke hinausgehender Fragestellungen - wie beispielsweise jene nach kontextuellen oder individuellen Handlungsstrategien, nach Aspekten von Hetero- und Plurilokalität sowie nach potenzieller Rückkehrmigration als einer neuen Form der Resilienz.

Wir verfolgen in diesem Beitrag mehrere Ziele: Zunächst stehen speziell die Varietät der neuartigen sozialen Praktiken sowie der multilokalen oder transregionalen Aktivitäten von Migranten und speziell die räumlichen Verflechtungen im Vordergrund. Ziel ist es, einen Beitrag zur Überwindung der beiden entscheidenden Schwachstellen der gegenwärtigen Migrationsforschung zu leisten - einerseits der nach wie vor bestehenden theoretischen Bias zwischen interner und internationaler Migration (KING et al. 2008; King \& SKeldon 2010; Vullnetari 2012) und andererseits der fehlenden Verknüpfung von Rational-Choice-Ansätzen mit Netzwerktheorien. Es geht mit anderen Worten um die notwendige Kombination von ökonomischen Determinismen und sozialen Migrationsnetzwerken, bei denen speziell Fragen der Inkorporation von Migranten in den Vordergrund gerückt werden (HAUg 2008). Mit Blick auf Rimessen und Rückkehrmigration konzentrieren wir uns dann auf Interdependenzen zwischen sozialer embeddedness und ökonomischer Bestimmtheit (SALAFF 2013, S. 462).

Solche Themen sind im Diskurs um das Transnationalismus-Konzept zwar behandelt worden, allerdings ist dort die Variabilität der räumlichen Maßstäblichkeit nur wenig berücksichtigt. Die Argumentation bewegt sich nach wie vor im Denkmuster nationaler „Container“ als räumlicher Entitäten. Deshalb wird im Folgenden der Blick nicht nur auf unterschiedliche Migrantengruppen (CASSARINO 2004, S. 270), sondern speziell auch auf die jeweiligen Wirkungsebenen (trans-national, trans-regional, trans-lokal) gerichtet. Mit dem vorgeschlagenen Konzept des Transregionalismus schließlich möchten wir die konzeptionelle Forschungsperspektive erweitern (vgl. Abb. 1), mit belastbarer Empirie präzisieren und so die Theoriedebatten um einen neuen Aspekt bereichern.

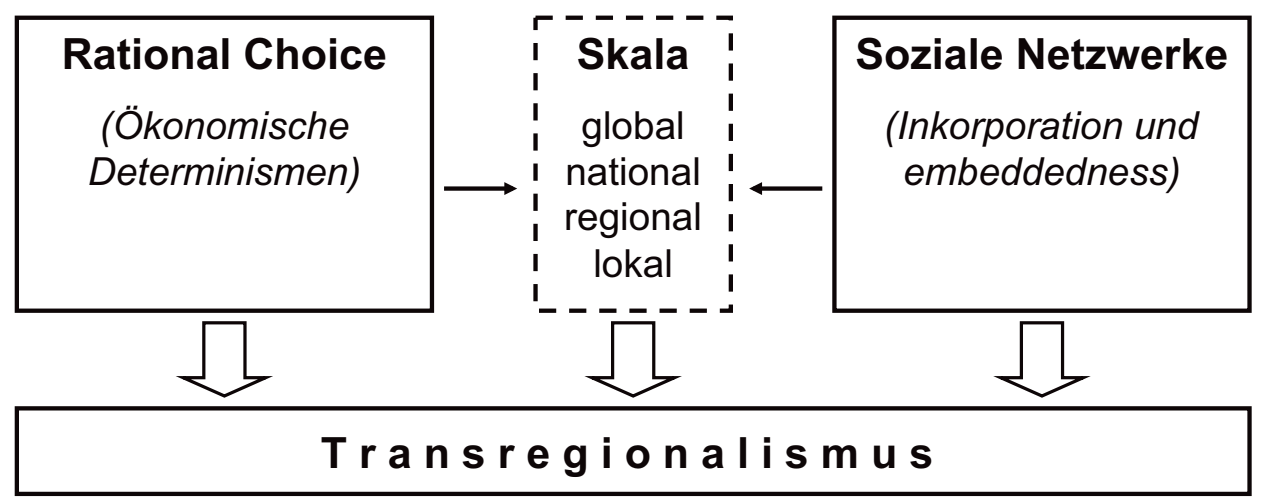

Abb. 1: Konzeptioneller Ansatz von Transregionalisierung 


\section{Von Transnationalismus zu Transregionalismus}

\subsection{Das Transnationalismuskonzept im Überblick}

Das Thema Transnationalismus bedeutete für die internationale Migrationsforschung einen neuen theoretischen Impetus. Dieses Konzept stellt weniger den eigentlichen Migrationsvorgang, als vielmehr den daraus resultierenden ,transnationalen sozialen Raum“ (Pries 2001) in den Mittelpunkt der Analyse. Ein solcher spannt sich zwischen den Herkunfts- und Zielgebieten oder der Sende- und Aufnahmegesellschaft auf, wenn Migration nicht als eindimensionale, endgültige Bewegung im Raum aufgefasst wird. Zentrales Element sind die sozialen Interaktionen zwischen beiden räumlichen Entitäten. Sie können das Ergebnis intensiver Kommunikation oder der Abfolge zirkulär-oszillierender Migrationen sein. Darin kommt sozialen Netzwerken und akteursbezogenen Netzwerkansätzen eine herausgehobene Rolle zu. Akteur und Netzwerk sind sedimentierte Manifestationen ihrer historischen und gegenwartsbezogenen sozialen Kontextualität: Die zugrunde liegende Entscheidung, die Motive und das angestrebte Ziel der Migration sind Ergebnis einer individuellen Biographie. Ebenso ereignet sich die Migration dann eingebettet in einen durch normative Komponenten definierten Rahmen.

\subsection{Mobilisierung und Transnationalisierung}

Mit solchen Überlegungen reagierte die Migrationsforschung auf die fundamentalen Veränderungsprozesse ihres Objektes. Die United Nations Population Division (2013) beispielsweise registriert für 2010 weltweit ca. 214 Mio. internationale Wanderungen, das heißt dass mehr als $3 \%$ der Weltbevölkerung pro Jahr den Wohnstandort zwischen Staaten wechseln - mit zunehmender Tendenz. Daneben dürfen die ca. 750 Mio. internen Migranten (Binnenwanderungen) nicht vergessen werden. Zu den hauptsächlichen Beweggründen der internationalen Migranten zählen die Suche nach Arbeit, Einkommen und Wohlstandsgewinn, aber auch die persönliche Sicherheit. Häufig handelt es sich um vulnerable Personen und Gruppen, welche ihr destabilisiertes Lebensumfeld verlassen (müssen). In der Zunahme der Mobilisierung und Transnationalisierung spiegeln sich neben Globalisierungseffekten auch post-sozialistische Transformationsprozesse wider.

Eine wesentliche Folge dieser Massenbewegung sind Geldüberweisungen von Migranten in ihre Heimatländer oder Herkunftsregionen. Sie lagen im Jahr 2011 erstmals über der 500-Mrd.-USD-Marke (516 Mrd. USD, WorLd BANK 2013). Allen Unkenrufen zum Trotz nimmt der Betrag der Rücküberweisungen also nach wie vor zu, trotz (oder gerade wegen?) der globalen Krise und den daraus resultierenden Schwierigkeiten für die Migranten (vgl. dazu auch RIESTER 2012). In vielen Fällen sichert der monetäre Transfer das Auskommen der Familien zu Hause. Er substituiert die dort fehlenden sozialen Sicherungssysteme und ist folglich Ausdruck sozialer Resilienz.

Im Hinblick auf die Frage von Migration und Entwicklung in der Sendegesellschaft vielleicht noch bedeutsamer sind ,social remittances“ (LEVITT 1999), also der Transfer 
von Humankapital, Innovation, Wissen und Kreativität. Derartige Faktoren werden häufig im Rahmen selbstständigen Unternehmertums volkswirtschaftlich in Wert gesetzt. Solche und andere Formen des brain gain bilden den positivistischen Kontrapunkt zum Phänomen des brain drain, welches die Wahrnehmung lang dominiert hatte. Dieser Aspekt, präzisiert durch die Frage nach dem Migrations-Entwicklungs-Nexus (FAIST 2008; HaAs 2010), findet seine theoretische Würdigung letztlich im Konzept des transnationalen sozialen Raumes. Dessen Implikationen bewegen sich, speziell bezogen auf Transformationszusammenhänge, zwischen Zwang und Ermöglichung und richten den Fokus mehr und mehr auf die wechselseitigen Abhängigkeiten. Gleichzeitig vernachlässigen sie jedoch zentrale Elemente räumlicher Diversität.

\subsection{Transnationalismus oder Transregionalismus?}

Die Transnationalisierung von Migrationen und insbesondere die gesellschaftlichen Folgen wurden in der vergangenen Dekade intensiv diskutiert (vgl. SMith \& GuARnizo 1998; Portes et al. 1999; Portes 2001, 2003; Kivisto 2001; Levitt 2001a; Vertovec 2001; Levitt et al. 2003; Levitt \& Jarowska 2007 oder Dunn 2010). Dabei wird insbesondere die neue Sichtweise auf ein soziales Phänomen betont, dessen Grundzüge genau genommen schon Elisabeth Lichtenberger (1984) im Zusammenhang mit der jugoslawischen Gastarbeiterwanderung als das „Leben in zwei Gesellschaften“ erkannt hatte. Zweifelsohne haben aber die transnationalen Verflechtungen mit den neuen Transport- und Kommunikationstechnologien und durch die zunehmende Mobilität insgesamt erheblich an Intensität, Spontaneität und Simultanität zugenommen (DAhinden 2009; Foner 2000; Vertovec 2004a).

Für eine Modellierung bietet sich der Vorschlag von Vertovec (2009) an. Hier wird die Varietät der Befunde bezüglich der Beobachtungsebenen, ihrer Funktionsmodi oder der konkret verorteten Folgen von Transnationalismus jeweils zwischen Begriffspaaren abgebildet: Transnationalismus from above und from below charakterisiert darin den Einfluss von Machtverhältnissen (Sмітн \& GUARNizo 1998), narrow und broad (ItZIGSOHN et al. 1999) bezieht sich auf den zum Beispiel durch institutionelle Vorgaben begrenzten Handlungsspielraum, core und expanded dagegen stellt eng gesetzten und überschaubaren, begrenzten Mustern den Fächer weit gespannter sozialer Gelegenheitspraktiken gegenüber (LevitT 2001a, b). Eine solche Typologie manifestiert sich - ungeachtet der Kritik von Faist (2013, S. 457) - zwischen unterschiedlichen Gruppen, wobei geographische Nähe oder Ferne und die Entwicklungsgeschichte (z.B. durch postkoloniale Einflüsse) eine elementare Rolle spielen können. Eine andere Zugangsperspektive ist der Fokus auf die Migranten selbst; schließlich führen Migranten räumlich getrennte Entitäten zu einer gemeinsamen Arena sozialer Aktion zusammen, indem sie sich vor- und rückwärtsgerichtet zwischen unterschiedlichen sozio-kulturellen und politisch-ökonomischen Systemen bewegen (FAIST 2000; PrIES 2008; Vertovec 2009; Dahinden 2009).

Trotz der unterschiedlichen Zugangsperspektiven besteht über zentrale Sachverhalte des Transnationalismus-Konzeptes Konsens. Das betrifft insbesondere die grundsätz- 
liche Variabilität des transnationalen sozialen Raumes und die Einbettung der Akteure in mehr als einen gesellschaftlichen oder nationalstaatlichen Bezug (LEvitT \& GLICK Schiller 2004; Pries 2005; Smith 2005). Die Arbeiten von Basch, Glick Schiller \& Blanc-Szanton (1994) oder Glick Schiller $(2003$, 2004, 2005) deuten aber bereits an, dass sich die Verlinkung innerhalb des transnationalen sozialen Raumes nur bedingt in nationalstaatlichen Dimensionen nachzeichnen lässt, sondern letztlich zwischen räumlich separierten Institutionen verläuft, welche manchmal unterschiedliche nationalstaatliche Bezüge aufweisen können (aber nicht müssen). In diesem Sinne bereicherte DunN (2010) die Debatte mit dem Hinweis, dass relevante Bindeglieder auch unterhalb der nationalstaatlichen Ebene verankert sein können. Sie finden sich zum Beispiel auf der Ebene von Familie zu Familie oder von Dorf zu Dorf. Insofern wäre dann der Begriff translokal sicherlich präziser (W ALDINGER \& FitZGERALD 2004, S. 1182; Velayutham \& Wise 2005; Müller-Mahn 2011). Mit diesem Argument erweitern wir im Folgenden die transnationale Perspektive um eine transregionale Komponente und gewichten damit - speziell hinsichtlich der räumlichen Folgen in Senderegionen - den geographischen Maßstab stärker (ähnlich BRENNER 1999; Herod \& Wright 2002; Marston, Hohn \& WoOdward 2005).

\subsection{Transregionalismus - Diversität der Migration als konstituierendes Element}

Die wechselseitigen Einflüsse durch zirkuläre oder oszillierende (sequenzielle) Migration sowie durch intensive Kommunikation mit entsprechenden Informations- und Erfahrungsaustauschen sind wesentliche räumliche Implikationen im transnationalen sozialen Raum. Dieses Muster ist - die empirischen Beispiele werden das zeigen äußerst differenziert. Es bedarf, gerade aus geographischer Sicht, der Erweiterung um die transregionale und mithin die relationale Perspektive. Im Gegensatz zu BeTTs' „Trans-regionalism“ (2011, S. 29) begreifen wir den Gegenstand nicht vor dem institutionellen Hintergrund oder aus Sicht der Governance, sondern bevorzugen, ganz ähnlich wie HoERder (2012), den räumlichen Zugang. Wir präzisieren das Konzept in Bezug auf Differenzierungen der Sendegesellschaft als fehlendes Bindeglied zwischen interner und internationaler Migration (vgl. Abb. 2). Dazu werden speziell die der Emigration manchmal vorgeschalteten oder einer Remigration folgenden Verlagerungsprozesse berücksichtigt. Das Spannungsfeld von Zirkularität und Rückkehr sowie die materiellen und immateriellen Austausche schaffen ein Wirkungsgefüge, dessen räumlich polarisierende Diversität im Kontext der Herkunft stärker zu gewichten ist.

Daraus lassen sich drei Thesen formulieren: (a) Transnationalisierung beinhaltet unterhalb der nationalen Ebene kleinräumige Differenzierungen; (b) entsprechende transregionale Netzwerke entfalten eine räumlich polarisierende Wirkung; (c) diese ist Ausdruck exogener, migrationsbasierter Handlungsstrategien. 


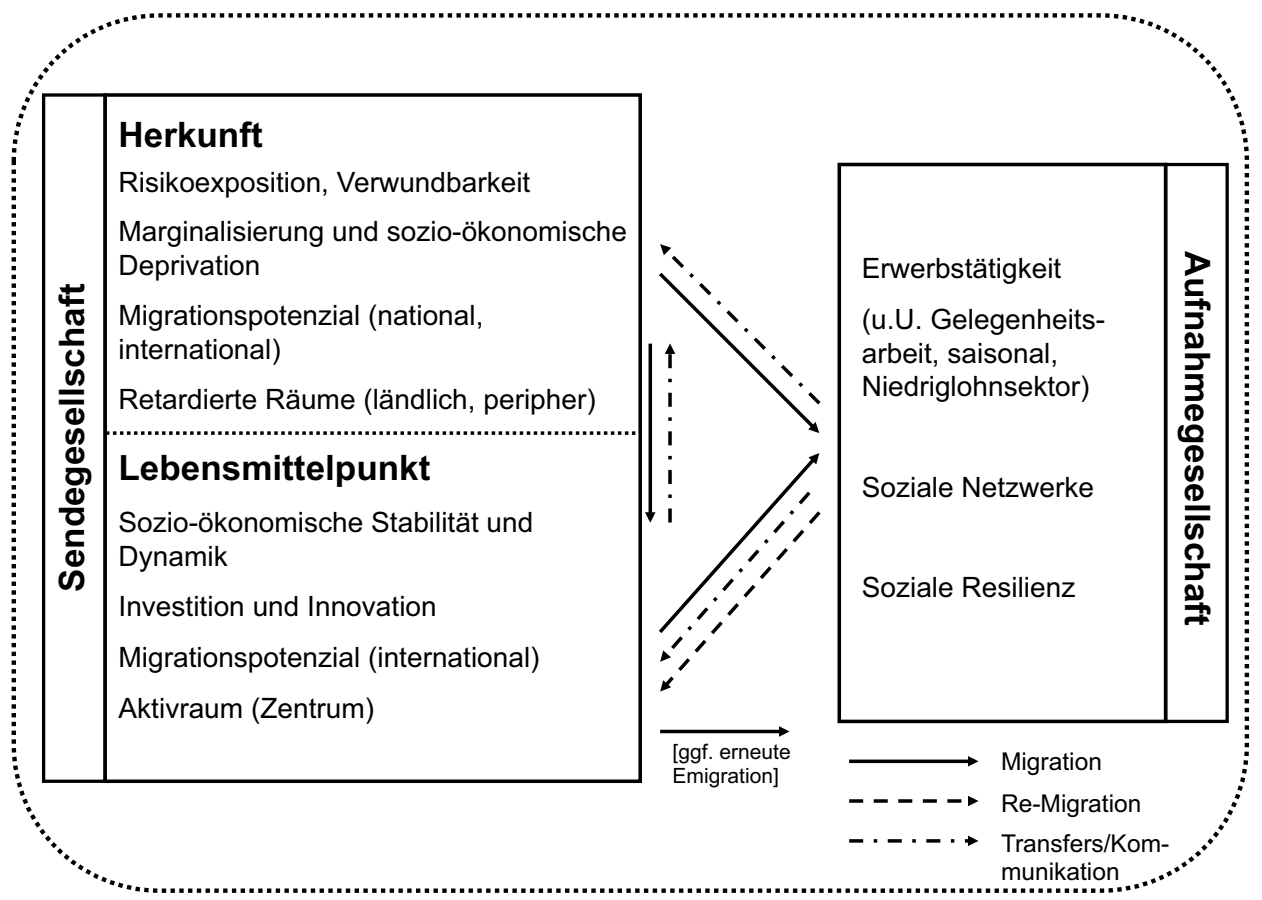

Abb. 2: Räumliche Implikationen von Transregionalismus: Konturen einer transregionalen Lebenswelt (in Anlehnung an GLick Schiller et al. 1992; Pries 2001; MülLER-Mahn 2011, S. 774)

\section{Diversität der Handlungskontexte - eine Annäherung an die Beispiele}

\subsection{9+: Transformation und Migration}

Mobilität und Migration in den sozialistischen Ländern waren teilweise eingeschränkt und mancherorts sogar stark reglementiert. Der Eintritt in die Transformationsphase brachte eine erhebliche Mobilisierung der Bevölkerung mit sich: Zu erinnern wäre an die innerdeutsche Migration Ende 1989, an die spontanen Fluchtbewegungen aus Albanien 1990/91, an die Zuwanderung aus GUS-Staaten in die Russländische Föderation in den 1990ern oder die privilegierte Migration von Spätaussiedlern aus der ehemaligen Sowjetunion nach Deutschland (vgl. z.B. FAssmann \& MüNZ 2000; Friedrich \& Schultz 2008; KING 2002).

In der Regel stabilisierten sich die Migrationssysteme in der Folgezeit. Nicht zuletzt vor dem Hintergrund der EU-Erweiterungen verblieb in Osteuropa eine anhaltende 
Ost-West-Arbeitsmigration als Konstante (FAssmann \& MünZ 2000), während für einige Länder Südosteuropas neben der Emigration unterschiedliche Formen sequenzieller Migrationen (zirkuläre, stepwise oder back-and-forth-Migrationen) bis heute charakteristisch sind.

\subsection{8+: Auswirkungen der globalen Finanz- und Wirtschaftskrise}

Die ersten beiden Dekaden post-sozialistischer Transformationen in Europa verliefen in einem - im Großen und Ganzen - günstigen weltwirtschaftlichen Umfeld, also in einem Agens einer vorwiegend exogenen Wachstumsdynamik. Die jeweiligen Transformationskrisen mit Währungsverfall, De-Industrialisierung oder politischen Instabilitäten bis hin zu kriegerischen Konflikten waren überwiegend endogenen Problemen zuzuschreiben. Insofern ist die Finanz- und Wirtschaftskrise seit 2008 die Nagelprobe hinsichtlich des Stabilitätsniveaus der Transformationsökonomien. Dabei stellt sich die Frage der generellen Resistenz der nationalen ökonomischen Systeme gegenüber einem abermaligen, nun jedoch extern verursachten Schockereignis. Gerade die baltischen Staaten zeigten sich nach einer dynamischen Entwicklungsphase mit teilweise zweistelligen Zuwachsraten im Bruttoinland sprodukt (BIP) im Jahr 2009 als besonders anfällig. Lettland beispielsweise hatte einen Rückgang der Wirtschaftsleistung um 17,7\% zu verzeichnen; in Litauen waren es $-14,8 \%$ und in Estland $-14,3 \%$ (Zahlen nach Global FinANCE 2013). Die in den 2000er Jahren fast schon überhitzte Dynamik und der rasche Wohlstandszuwachs hatten in Lettland zahlreiche Privatanleger möglicherweise zu riskanten Anlagen verleitet.

Im Transformationszusammenhang des sogenannten Westbalkans, wo die Wirtschaftsleistung insgesamt deutlich niedriger ist und sich vor allem anfänglich ein geringeres Wachstum entfalten konnte, fiel der Einbruch weniger gravierend aus. Albanien beispielsweise hatte im Krisenjahr 2009 immer noch einen BIP-Zuwachs von 3,3\% (Global Finance 2013). Die Gründe dürften im jeweiligen Grad der Verflechtung mit der Weltwirtschaft und speziell den internationalen Finanzmärkten zu suchen sein.

Beiden Kontexten (baltische Staaten und Westbalkan) gemein ist der stabilisierende Effekt der Emigration, die mit Berechtigung als Entwicklungsfaktor interpretiert werden kann. Migration entlastete einerseits den heimischen Arbeitsmarkt; andererseits resultierten aus monetären und sozialen Rimessen sowie migrationsbasiertem Unternehmertum direkte volkswirtschaftliche Impulse.

Neben der ökonomischen Resistenz sind für die Gesellschaften und ihre Migrationssysteme vor allem Fragen und Formen sozialer Resilienz entscheidend. Die Feststellung, dass ,Trotz der Wirtschaftskrise [...] von Migranten 2012 mehr Geld in ihre Herkunftsländer als je zuvor“ überwiesen wird (PAPE 2012, S. 7; Hervorhebung durch die Verfasser) ist im Grunde falsch formuliert, denn Rimessen zeigten sich als sehr robust gegenüber der Krise. Fallweise wird offenbar wegen der Krise sogar mehr in die Heimat transferiert - so zum Beispiel zur Vorbereitung einer anstehenden Rückkehr. Selbst die These, dass Arbeitsmigranten vom verlangsamten Wachstum der Weltwirtschaft generell betroffen sind, lässt sich so pauschal nicht halten (dazu auch 
Hillmann et al. 2012, S. 3f.). Es zeigte sich zwar, dass Migranten vor allem in besonders krisenanfälligen Arbeitsmarktsegmenten wie der Bauwirtschaft überproportional betroffen waren, im Falle der wirtschaftlichen Erholung und nicht zuletzt wegen ihrer Flexibilität aber rascher wieder eine Beschäftigung fanden. Insofern sollte das Thema Krisenmigration nach Migrationsformen segmentiert und nach regionalen Kontexten differenziert bewertet werden.

\section{Transformationspfade und Migrationssysteme}

\subsection{Anmerkungen zur Methodologie}

Die differenzierende Analyse der Veränderlichkeit unterschiedlicher Migrationssysteme basiert auf empirischen Befunden aus Lettland und Albanien und folgt dem Motiv der „Vergleichbarkeit des Unvergleichbaren“. Die Konzeption und Methodik ist den jeweiligen Umständen angepasst und bewegt sich zwischen Ansprüchen der Repräsentativität durch große Stichproben einerseits und Authentizität durch Narration andererseits.

Der Befund für das lettische Beispiel stützt sich auf umfangreiche webbasierte Untersuchungen über das soziale Netzwerk www.draugiem.lv. Mit Zustimmung des Betreibers wurde dort ein Fragebogen mit Angaben zur soziodemographischen Struktur, zu Migrationsmotiven und Migrationserfahrungen eingestellt, der von den Nutzern online ausgefüllt werden konnte. So konnten im März 2012 lettische Migranten im Vereinigten Königreich $(n=1117)$, in Deutschland $(n=426)$, Irland $(n=618)$ sowie in Schweden und Norwegen $(n=404)$ erreicht werden. Die Daten für 2010 stammen aus einer Studie, die mit gleicher Methodik im Vereinigten Königreich durchgeführt worden war. Altersstruktur und Geschlechterproportion des Samples entsprechen denen der Nutzer des Portals, in dem insgesamt $87,6 \%$ der lettischen Internetnutzer vertreten sind (Click H+K Research 2012). 59,8\% gaben an, die Plattform täglich zu nutzen (vgl. Apsite-Berina 2013). Die Respondenten der Surveys im Jahr 2010 und 2012 sind zwischen 16 und 63 bzw. 16 und 71 Jahre alt, wobei die Mehrzahl zur Altersgruppe der unter 30-Jährigen gehört.

Der albanische Fall wurde mit qualitativ-narrativen Methoden beforscht. Hier konnten die Erkenntnisse aus 40 Tiefeninterviews zum Thema Rückkehrmigration vom Oktober 2012 mit Ergebnissen einer Befragung mit ähnlicher Thematik aus dem Jahr 2006 (n=34 zu Migration und Unternehmertum, dazu GöLER 2007) verglichen werden. Der Ansatz nutzt insbesondere die Auswertung von Migrationsbiographien (vgl. CARLing 2012, S. 149; Beispiele bei KIng et al. 2008).

Die methodische Unterschiedlichkeit erscheint insofern vertretbar, da weder die jeweiligen Erhebungen noch individuelle Fälle daraus miteinander in Beziehung gesetzt werden. Es werden lediglich zentrale Ergebnisse synthetisch zusammengeführt, um den komplementären Charakter der beiden empirischen Studien in Wert zu setzen. 


\subsection{Das lettische Beispiel}

Der wirtschaftliche Transformationsprozess in Lettland verlief schneller als in anderen ehemaligen Sowjetrepubliken. Insbesondere der tertiäre Sektor entwickelte sich rasch. Nach dem EU-Beitritt im Jahr 2004 wurden in vier aufeinanderfolgenden Jahren volkswirtschaftliche Zuwachsraten von mehr als $10 \%$ erreicht. Der Kapitalzufluss aus dem Ausland führte, angeheizt durch niedrige Zinsen und den Markteintritt ausländischer Banken, zu einem Anwachsen des Darlehens- und Hypothekenmarktes. Der Bevölkerungsrückgang durch rückläufige Fertilität wurde durch Auswanderung verstärkt. Dazu gehört neben der Rückwanderung ethnischer Russen nach der lettischen Unabhängigkeit 1991 speziell die bis in die Gegenwart hochwirksame Arbeitsmigration. Lettland hat sich in dieser Zeit vom Einwanderungs- zum Auswanderungsland gewandelt.

Grundsätzlich lassen sich drei Phasen unterschiedlicher Migrationsregime abgrenzen: das reglementierte System der Sowjetunion, die Massenmigrationen zwischen Unabhängigkeit und EU-Beitritt sowie die Phase der Annäherung an das EU-Migrationssystem (KRIŠJĀNE et al. 2009). Mit Einsetzen der Transformationsprozesse veränderten sich dabei neben den Motiven sowohl die Richtung als auch die Intensität. Die enge Anbindung an den (post-)sowjetischen Raum wurde von einer ausgeprägten Orientierung an Westeuropa abgelöst. Spätestens mit dem Zugang zum Arbeitsmarkt der etablierten EU-Länder war die Umkehr zur Sendegesellschaft vollzogen. Der massive ,young drain“ (KRIŠJĀNE 2008, S. 69) durch die Abwanderung der aktiven und qualifizierten Bevölkerung im arbeitsfähigen Alter schwächt, wie in den anderen baltischen Staaten auch, den heimischen Arbeitsmarkt.

Von den demographischen und ökonomischen Komponenten dieses Wandels sind nicht zuletzt die ländlichen Regionen betroffen, welche unter einer massiven Depopulation mit Alterungsprozessen leiden. Die Entwertung der Peripherie hängt mit Auswanderung und, ganz erheblich, mit peripher-zentralen Binnenmigrationen zusammen. Die Klein- und Mittelzentren können mit der Attraktivität der Agglomeration der Hauptstadt Riga [Rīga] nicht Schritt halten, insbesondere was das Angebot an Ausbildungsmöglichkeiten betrifft. So ist die Hälfte der jungen Bevölkerung Lettlands in Riga konzentriert.

Soziale Probleme, hohe Arbeitslosigkeit, fehlende Erwerbsalternativen und die Konsequenzen der demographischen Krise sind Implikationen des Migrationswandels nach 1991, der sowohl auf dem Lande wie in der Stadt einschneidend war. In diesem Wirkungsgefüge bleiben die ländliche Peripherie, Klein- und Mittelstädte sowie die metropolitane Region Riga durch Kettenmigrationen netzwerkartig verbunden (vgl. z.B. KRIŠJĀne 2007; Eglīte et al. 2006). Erst neuerdings (nach 2004) führen Migrationen vermehrt wieder direkt ins Ausland.

\subsubsection{Migration im Zeichen von EU-Beitritt und Finanzkrise}

Im Vergleich der beiden Etappen vom EU-Beitritt 2004 bis zum Beginn der Wirtschaftskrise und der Zeit während der Krise ab 2010 hat sich die strukturelle Zusammensetzung der lettischen Migranten signifikant verändert. Das betrifft zunächst - am 
Beispiel der Migranten in das Vereinigte Königreich - vor allem geschlechtsspezifische Differenzierungen, nach denen sich der Anteil männlicher Migranten nach 2010 massiv vergrößert hat. Vor der Krise waren zudem Migrationen im Familienverbund deutlich häufiger vertreten. Das korrespondiert mit dem zunehmenden Anteil an Personen, die vor der Emigration in Lettland gearbeitet hatten, dann jedoch wegen der Verschlechterung der Bedingungen zur Emigration mehr oder weniger gezwungen waren. Zudem hat sich der Anteil junger Migranten erhöht, was letztlich mit den Problemen von (Hoch-) Schulabgängern auf dem lettischen Arbeitsmarkt zusammenhängt. Ältere Migranten dagegen sind vor allem durch höhere Löhne im Ausland motiviert.

Die Untersuchungen unter lettischen Migranten (vgl. Tab. 1) zeigen deutlich, dass sich die treibende Kraft der Emigration vom Erreichen eines höheren Lebensstandards (im Ausland) hin zum Bewahren des Lebensstandards (zu Hause) gewandelt hat. Auffällig ist die Zunahme des Motivs finanzieller Probleme und Kredite bei den „Krisenmigranten“. Generell überwiegen bei männlichen Migranten die materiellen Aspekte, während Frauen signifikant häufiger familiäre Gründe nennen. Im Jahr 2012 wurden als die drei Hauptgründe finanzielle Probleme im Zusammenhang mit Immobilienkrediten, die unsichere Perspektive in Lettland sowie Arbeitslosigkeit genannt (vgl. Tab. 2). Analog dazu hat der Wert der Rücküberweisungen, die in Lettland etwa $2 \%$ des BIP ausmachen, zwischen 2009 und 2012 um 23,9\% zugenommen (von 591 Mio. USD auf 732 Mio. USD; 2000 lag der Wert noch bei 72 Mio. USD; WorLd B ANK 2013).

Es besteht ein enger Zusammenhang mit der lettischen „Immobilienblase“. Unterstützt durch das niedrige Zinsniveau während der Phase der Hochkonjunktur, welche sich auch in hohen Löhnen ausdrückte, wurden in großem Umfang Darlehen aufgenommen, häufig zur Finanzierung einer Wohnung, bisweilen auch Konsumentenkredite. Dieses Wohlstandsphänomen war vor allem in der boomenden Hauptstadt Riga einschließlich des suburbanen Raumes (dem bevorzugten Zielgebiet der zuvor aus dem übrigen Land Zugewanderten) verbreitet, weniger in den ländlichen und peripheren Regionen. So wird plausibel, dass im Rahmen der gegenwärtigen „Krisenmigration“ der Anteil der Migranten aus dem Großraum Riga, der nun überproportional gut qualifizierte Arbeitskräfte verliert, sehr hoch ist. Hervorzuheben ist, dass viele der neuen Migranten bislang keine Migrationserfahrung hatten.

In den Befragungen finden sich wiederholt Hinweise auf die Verbindungen von hohen regelmäßigen Ratenzahlungen in Kombination mit Lohnkürzungen oder Arbeitsplatzverlust, das heißt es besteht ein kausaler Zusammenhang zwischen Rezession und drohender Überschuldung. Im vorliegenden Fall ist die Migration also eine Reaktion auf eine mehr oder weniger große existenzielle Notlage und mithin ein resilienter Mechanismus. Folgerichtig ist bei den Migranten der letzten Jahre die Bereitschaft zur Rückkehr deutlich höher als dies bei den frühen Migranten der Fall war (APSITE-BERINA 2013).

Aus dem lettischen Beispiel können drei Schlüsse gezogen werden: (1) Die Krise bedingte in Lettland eine neuerliche Auswanderungswelle. (2) Unter den früheren Migranten dominieren die, welche im Ausland geblieben sind (APsite et al. 2012). (3) In räumlich differenzierender Sicht ist der suburbane Raum der Agglomeration Riga besonders betroffen. 


\begin{tabular}{|l|c|c|c|c|c|c|}
\hline \multirow{2}{*}{$\begin{array}{l}\text { Motive der Emigration } \\
\text { Angaben jeweils in \% } \\
\text { aller Befragten) }\end{array}$} & \multicolumn{3}{|c|}{$\begin{array}{c}\text { Emigration 2004-2007 } \\
\text { (nach EU-Beitritt) }\end{array}$} & \multicolumn{2}{|c|}{$\begin{array}{c}\text { Emigration 2008-2010 } \\
\text { (,Krisenmigranten“) }\end{array}$} \\
\cline { 2 - 8 } & Männer & Frauen & Gesamt & Männer & Frauen & Gesamt \\
\hline Arbeitslosigkeit & 29,9 & 16,9 & 20,1 & 49,1 & 37,2 & 41,5 \\
\hline Höhere Einkommen & 42,5 & 46,7 & 45,6 & 54,5 & 51,8 & 52,8 \\
\hline Bessere Lebensbedingungen & 57,5 & 54,0 & 54,7 & 54,5 & 51,0 & 52,3 \\
\hline Finanzielle Probleme und Kredite & 11,5 & 8,4 & 9,2 & 29,5 & 23,6 & 25,7 \\
\hline Reise und Entdeckung & 19,5 & 12,3 & 14,0 & 22,3 & 14,9 & 17,6 \\
\hline Sprachkenntnisse verbessern & 20,7 & 16,1 & 17,2 & 29,1 & 29,3 & 29,2 \\
\hline Familiäre Gründe & 23,0 & 22,6 & 26,1 & 17,7 & 22,0 & 22,8 \\
\hline Sonstiges & 4,8 & 4,3 & 4,5 & 3,3 & 4,5 & 4,0 \\
\hline Zahl der Respondenten & \multicolumn{7}{|c|}{$\mathrm{n}=349$} & \multicolumn{5}{c|}{$\mathrm{n}=602$} \\
\hline
\end{tabular}

Datenquelle: Online-Survey 2010

Tab. 1: Motive für die Emigration in das Vereinigte Königreich

\begin{tabular}{|l|c|c|c|}
\hline $\begin{array}{l}\text { Motive der Emigration } \\
\text { (Angaben jeweils in \% aller Befragten) }\end{array}$ & Männer & Frauen & Gesamt \\
\hline Finanzielle Probleme, Immobilienkredite & 46,4 & 41,3 & 43,4 \\
\hline Unsichere persönliche Zukunftsperspektive & 48,7 & 37,5 & 42,1 \\
\hline Arbeitslosigkeit & 43,4 & 35,1 & 38,5 \\
\hline Persönliche Karriere & 40,1 & 27,5 & 32,6 \\
\hline Familiäre Gründe & 17,8 & 32,8 & 25,5 \\
\hline Reise und Entdeckung & 18,5 & 18,5 & 18,5 \\
\hline Mängel des lettischen (Aus-)Bildungssystems & 6,2 & 5,8 & 6,0 \\
\hline Sonstiges & 9,4 & 9,7 & 9,8 \\
\hline
\end{tabular}

Datenquelle: Online-Survey 2012; $\mathrm{n}=2565$

Tab. 2: Motive der Emigration lettischer Migranten in Zeiten der Krise

\subsection{Das albanische Beispiel}

Die albanische Gesellschaft ist eine Migrationsgesellschaft. Emigration zieht sich - mit Ausnahme der sozialistischen Ära, in der Außenwanderung fast vollständig unterbunden und Binnenmigration stark reglementiert war - wie ein roter Faden durch die Geschichte. So ist es nur folgerichtig, dass es unmittelbar nach der Öffnung des Landes ab 1990 zur Massenmigration kam (BARJABA \& KING 2005; VullnEtARI 2012), zu Binnenwanderungen ebenso wie zu internationalen Migrationen (GöLER 2009). Im Jahr 2010 verfügte fast die Hälfte der Bevölkerung über persönliche Migrationserfahrung 
(45,4\%; World B ANK 2011, S. 54). Nicht zuletzt hing die ökonomische Stabilisierung des Landes nach 2000 mit Wachstumsraten im BIP zwischen 5 und 10\% wesentlich mit Effekten der Migration zusammen.

\subsubsection{Etappen der jüngeren albanischen (E-)Migrationsgeschichte}

Bereits in den ersten Transformationsjahren bis 1999 hatten fast 750.000 Albaner das 3-Millionen-Einwohner-Land verlassen (Vullnetari 2007, S. 36), davon rund 500.000 nach Griechenland und 200.000 nach Italien. Im Jahr 2010 waren es 1,438 Mio. (World Bank 2011, S. 54). Migranten der frühen Phase waren oft junge, unverheiratete Männer, die - falls nötig - das Risiko illegaler Grenzübertritte und Arbeitsaufenthalte auf sich nahmen. Die Migranten in den angrenzenden Mittelmeerraum waren in der Regel geringer qualifiziert als jene, die dauerhaft in die USA oder nach Kanada auswanderten. Die anfangs typische back-and-forth-Arbeitsmigration bekam nach 1996/97 verstärkt dauerhafte Züge, als Unruhen den albanischen Transformationspfad unterbrachen und eine massive Destabilisierung im Land bedeuteten. Migration im Familienverbund, Familiennachzug oder die Gründung einer Familie im Ausland signalisieren die sukzessive Verstetigung des Lebens außerhalb der Heimat.

Daneben besteht nach wie vor eine starke Land-Stadt-Migration. Tiranas [Tiranë] Einwohnerzahl hat sich durch 20 Jahre Zuwanderung aus der Peripherie des Landes verdreifacht. Viele der Migranten blieben dennoch ihren Heimatdörfern verbunden sei es, dass die ältere Generation noch dort lebt, sei es, dass dort Grundbesitz besteht. Häufig ist die Hauptstadt das Sprungbrett zur Emigration gewesen, sodass das Leben vieler Familien - als Folge mehrfacher Migrationen - heute fast paradigmatisch transregional im Sinne von Abbildung 2 konstituiert ist.

\subsubsection{Remittances, Rückkehrmigration und migrantische Ersparnisse}

Geldüberweisungen und Sachleistungen von Migranten (Rimessen; engl. remittances) sicherten in der schwierigen Transformationsperiode zahllosen Familien das Überleben und stellen nicht selten die Basis eines gewissen Wohlstands dar. 70\% der Migranten senden Rimessen. Das Gesamtvolumen stieg von ca. 400 Mio. USD (1994; DeZwager et al. 2005, S. 21) auf den bisherigen Höchstwert von knapp 1,5 Mrd. USD im Jahr 2008 (WoRLd B ANK 2011, S. 54). Zeitweilig war das ein Fünftel des gesamten albanischen BIPs - mehr als der Wert der industriellen Produktion und mehr als die ausländischen Direktinvestitionen (DEZwAGER et al. 2005, S. 21). Schätzungen für 2010 beliefen sich auf 1,285 Mrd. USD (WoRLD B ANK 2011, S. 54), was immer noch einem BIP-Anteil von $13,7 \%$ entspricht. Im Zeichen der Krise ist der private Kapitalzufluss nach Albanien rückläufig.

Die Verausgabung der Finanzmittel ist offenbar vom regionalen, sektoralen und individuellen Zusammenhang abhängig. Zwar sind Rimessen nicht zwingend ein differenzierendes Kriterium für Wohlstand oder Armut (ARREHAG et al. 2005), wohl aber machen sie einen erheblichen Teil der Privatbudgets aus und ,there is a corresponding risk of an over-reliance on remittances“ (KInG et al. 2011a, S. 277). MüLLER \& MunRoE (2008) berichten zum Beispiel von einer ,rent seeking mentality“ im Agrarraum, das 
heißt dass landwirtschaftliche Tätigkeit zugunsten des Lebens von remittierten Mitteln aufgegeben wird. Rimessen verschärfen somit die regionalen Disparitäten. Während Rimessen in der Peripherie zur Extensivierung der Landnutzung führen können, führen sie anderswo zur Intensivierung der Geschäftstätigkeit. So stellt „Returnee’s Business“ (GöLER 2011) vor allem im städtischen Raum eine relevante Größe für den Arbeitsmarkt dar. Die Verflechtung zwischen Migration und Unternehmertum scheint in Albanien extrem. Die Studie zu Unternehmensgründungen im produzierenden Sektor (GöLER 2007) zeigte, dass in 33 von 34 untersuchten Fällen dem Gründungsvorgang die Emigration vorausgegangen war. Migration als gründungsförderndes Element beinhaltet zunächst die monetäre Seite. In Albanien werden ca. 40\% des unternehmerischen Startkapitals durch Arbeitsaufenthalte in der Emigration erwirtschaftet (UNDP 2000, S. 43). Nicht zu unterschätzen ist darüber hinaus der Technologie- und Innovationstransfer in Form von in der Emigration erworbenen Wissens. Insofern tritt zum allenthalben beklagten brain drain und brain waste auf diese Weise ein brain gain, wenn Migranten nach ihrer Rückkehr als selbstständige Unternehmer auftreten.

Die Biographie des Miteigentümers einer mittelständischen Möbelfabrikation in Vlorë (Südalbanien) mag das verdeutlichen. Der gelernte Ingenieur widmete sich um das Jahr 1992 dem Verkauf von Obst in einem Kiosk, ging zum Import von Lebensmitteln und später von Spanplatten über und gründete 1997 ein stationäres Einzelhandelsgeschäft. Da sich dieses als Misserfolg erwies, begann er 1999 wiederum mit dem Import von Möbelkleinteilen, bis 2003 die jetzige Firma gegründet wurde. In immer wieder zwischengeschalteten Auslandsaufenthalten, genannt werden Spanien, Dubai und Italien, verdingte sich der jetzige Unternehmer zumeist als Bauarbeiter. Typisch erscheinen, auch im Vergleich mit weiteren in der Studie erhobenen Biographien, der mehrfache Branchenwechsel und die Auslandsaufenthalte zur Kapitalbeschaffung oder -aufstockung. Allen zwischenzeitlichen Misserfolgen und den Risiken des praktizierten trial-and-error-Prinzips zum Trotz - bei einem abermaligen Besuch im Oktober 2012 stellte sich die Firma im zehnten Jahr ihrer Existenz als stabil dar.

Bisweilen gehen solche Rückkoppelungen weit über die Akkumulation von Kapital und Wissen hinaus: Einige albanische Rückwanderer hatten die für den Aufbau einer Produktion in Albanien notwendigen Maschinen aus der Emigration mitgebracht. Viele unterhalten Geschäftsbeziehungen zu ihren ehemaligen Arbeitgebern im Ausland.

Andere Befunde lassen eine zu positive Interpretation des returnees business allerdings nicht angezeigt erscheinen. So fiel in der Studie, die in einer Phase der albanischen Hochkonjunktur durchgeführt wurde, vor allem die ausgesprochene Ausbildungsferne der Gründungsaktivitäten auf. Nur in wenigen Fällen brachten die neuen Unternehmer formale Qualifikationsmerkmale in der jeweiligen Branche mit. In einigen Fällen waren die Gründungen völlig branchenfremd vollzogen worden.

\subsubsection{Von der Perspektiv- zur Krisenmigration?}

Die globale Krise nach 2008 lässt das vorstehend skizzierte (E-)Migrationsmuster nun in einem anderen Licht erscheinen. So sind zahllose Albaner in Griechenland, dem Hauptziel der Emigration, von der dortigen Krise voll erfasst worden. Für sie gilt das Motto „last hired, first fired“ ganz besonders (vgl. auch Gedeshi \& DeZwager 2012). 
Das hängt - neben der konjunkturellen Lage - mit der besonderen Risikoexposition und Verwundbarkeit der Migranten zusammen. 26\% der albanischen Erwerbstätigen in Griechenland sind ,informell“, faktisch also als Schwarzarbeiter, beschäftigt (DEUTSCHE Welle vom 8. November 2011; auch Gedeshi \& DeZwager 2012).

Während sich die Frage der Rückkehr für viele Emigranten aus anderen osteuropäischen Ländern (wie Lettland, s.o.) in der Regel nicht stellt, deutet sich im albanischen Migrationssystem eine teilweise Umkehr bislang bekannter Schemata an. Die eher erfolgsbetonte Rückkehrmigration während der politischen und ökonomischen Stabilisierung und in der Phase des Aufschwungs nach 2000 (NIKAS \& KING 2005; Labrianidis \& Hatziprokopiou 2005; Kilic et al. 2009; Germenji \& Milo 2009; King et al. 2011; GöLER 2011) wird nun von der jüngeren, kriseninduzierten Remigration um 2011/12 kontrastiert (Gedeshi \& DeZwager 2012; Göler \& DoKa 2013).

Die negativen Auswirkungen der Krise hängen primär mit den ökonomischen Problemen in den Hauptzielgebieten der Auswanderung der vergangenen beiden Dekaden zusammen. Zunächst schienen Albaner in Italien stärker betroffen, bis sich 2010 die Lage in Griechenland massiv verschlechterte. Das äußerte sich dort außer in der zunehmenden Arbeitslosigkeit in der Reduzierung der Arbeitsstunden, im Wechsel in unterqualifizierte Jobs und in entsprechenden Einkommenseinbußen. Sie werden für 2008 und $2009 \mathrm{im} \mathrm{Schnitt} \mathrm{mit} \mathrm{10,7 \%} \mathrm{beziffert,} \mathrm{was} \mathrm{sich} \mathrm{unter} \mathrm{anderem} \mathrm{in} \mathrm{einer}$ reduzierten Sparquote bemerkbar machte. Gedeshi \& DEZwager (2012, S. 247) nennen verschiedene Strategien der Problembewältigung, die zumeist (noch) als Bleibeoption formuliert wurden, wenngleich sich die Möglichkeit einer parziellen Rückführung der Familie oder die Rückkehr des gesamten Haushaltes bereits damals ${ }^{2}$ abzeichneten (Gedeshi \& DeZwager 2012, S. 250f.). In den vergangenen fünf Jahren sollen allein aus Griechenland 180.000 Albaner zurückgekehrt sein (ACIT 2012, S. ii); das wäre immerhin ein Fünftel aller Emigranten nach Griechenland - mit zunehmender Tendenz.

Zu den Rückkehrern zählt auch ein 50-jähriger „Multi-Entrepreneur“ aus Dhermi in Südalbanien, dessen Aussagen sich zwischen Erfolgsgeschichten und Fatalismus bewegen. In kommunistischer Zeit im Fernmeldewesen beschäftigt, nutzte er 1991 die Gelegenheit zur Emigration und fand in Athen [Athina] eine Beschäftigung in der Lebensmittelbranche. Neben dem zuletzt relativ guten Gehalt erhielt er von seinem letzten Arbeitgeber, der in wirtschaftliche Schwierigkeiten geraten war, im Jahr 2011 eine Kompensation. Die Familie ist in Griechenland in hohem Maße inkorporiert: Die Frau arbeitet als Kellnerin, der Sohn hat nach Universitätsabschluss eine Anstellung bei einer Bank gefunden und die zehnjährige Tochter geht zur Schule. Man hat es zu Wohneigentum gebracht. Nachdem sich die Situation in Griechenland verschlechtert hatte, kam 2011 der Entschluss zur parziellen Rückkehr: Die Frau bleibt in Athen, so lange sie noch beschäftigt ist. Man hofft, dass die Tochter die Schule dort beenden kann. Zwischenzeitlich baut der Mann in Dhermi eine auf Landwirtschaft basierte dörfliche Existenz auf (,mit Landwirtschaft hat man immer zu essen ...“), wobei klar ist, dass für die Nachkommen eine Rückkehr dorthin nicht in Frage kommt. Hier liegen die Optionen zwischen Bleiben in Griechenland (wo die Situation allerdings als

\footnotetext{
2) Die zitierte Studie repräsentiert den Stand von Ende 2009, also ein ganz frühes Stadium der albanischen
} Remigration. 
viel zu unsicher eingeschätzt wird), dem Wechsel ins westeuropäische Ausland oder, wenn nach Albanien, dann in die Hauptstadt.

Nicht nur in diesem Falle korrespondiert eine Rückkehr durchaus mit der längerfristigen Lebensplanung; das ist bei vielen Auslandsalbanern so (vgl. LabRianidis \& Hatziprokopiou 2005). Eine IOM-Studie bezifferte das entsprechende Migrationspotenzial auf 56\% (DEZwAGER et al. 2005, S. 21). Remigration ist gerade bei jenen im näheren europäischen Ausland als Handlungsoption ausdrücklich vorgesehen, das heißt das Leben im Ausland ist von Anfang an als eine Etappe in der individuellen Biographie geplant. Darin dient der Auslandsaufenthalt hauptsächlich dazu, in einer Übergangsphase die Familie in der Heimat zu versorgen, dort nach der Rückkehr eine gesicherte Existenz zu führen und den Nachkommen ein besseres Leben bieten zu können. Das Problem ist somit nicht unbedingt die Tatsache der Rückkehrmigration, sondern vor allem der falsche (weil zu frühe oder zu späte) Zeitpunkt der Rückkehr. Im einen Fall hat die Aufenthaltsdauer im Ausland eventuell noch nicht zur Kapitalakkumulation gereicht und im anderen steht die Rückkehr möglicherweise zu einem lebenszyklisch ungünstigen Zeitpunkt an. So ist bei zahlreichen Familien in der Wachstums- und Konsolidierungsphase die Integration der im Ausland geborenen Kinder problematisch. Allein in das südalbanische Gjirokastër sind mehr als 260 Kinder zurückgekehrt (VoICE of AMERICA/GJiROKAStËr am 15.2.2012), bei denen sich nun der Schulbesuch wegen der Sprachprobleme schwierig gestaltet. Für die nach-kommunistische Generation ist die Rückkehr in den allermeisten Fällen ohnehin keine echte Alternative oder Perspektive.

Bei einem jungen Mann, Jahrgang 1994 (vgl. Abb. 3), kann eine Art mentale lebensweltliche Bifurkation diagnostiziert werden: Der 18-Jährige hat bis zur Rückkehr im Jahr 2009 mit Ausnahme der ersten drei Monate sein ganzes Leben in Griechenland verbracht. Zwar hatten die Eltern den Zeitpunkt ihrer Rückkehr offenbar sorgfältig gewählt und sich in Sarandë sofort selbstständig gemacht (der Mann) bzw. dort eine Arbeit aufgenommen (die Frau). Selbst wenn der Sohn die weiterführende Schule absolviert und nun ein Studium an der Hochschule in Gjirokastër aufgenommen hat - es gelingt ihm nicht, einen glücklichen Eindruck zu vermitteln bezüglich des Lebens in einem Land, dessen Nationalität er zwar hat, das er aber kaum kennt: „I am not feeling Albanian, I feel Greek“ und „I cannot express myself in the Albanian language“. Angesichts solcher Aussagen ist verständlich, dass er die nächste Gelegenheit zur abermaligen Emigration nutzen will, trotz fehlender Jobs und Krise in Griechenland; momentan fehlen lediglich die Möglichkeiten dazu.

Viele Jugendliche drücken das ähnlich aus. Sie sehen die persönliche Zukunft im Ausland oder, wenn in Albanien, dann nicht im Dorf oder in den touristisch geprägten und nur saisonal vitalen Küstenorten, sondern in Tirana.

\subsubsection{Lektionen aus Albanien}

Waren bis vor kurzem die immensen Ersparnisse von im Ausland lebenden Albanern - verbunden mit der Erwartung, dass die Migranten bei der Rückkehr im Rahmen ihres durchschnittlich 17 bis 18 Jahre währenden „Migrationszyklus“ ihre Ersparnisse mitbringen - fast euphorisch gesehen worden (z.B. DEZwAGER et al. 2005), 


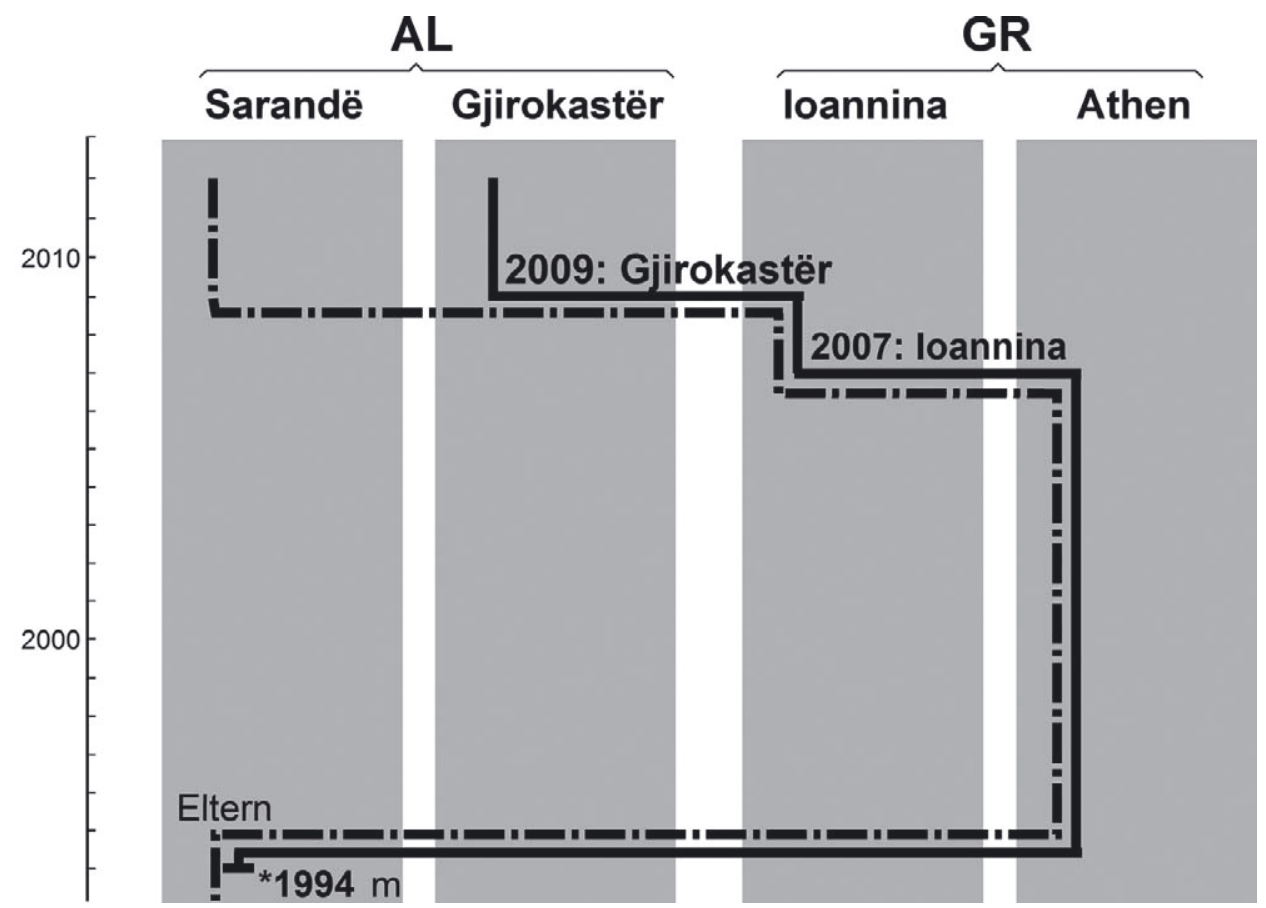

Abb. 3: Migrationsbiographie eines 18-Jährigen aus Sarandë in Südalbanien (Oktober 2012)

so ist diesbezüglich Ernüchterung eingetreten. Konnte eine Rückwanderung in den 2000er Jahren mit CERASE (1974) vielfach als return of success interpretiert werden, so scheint die Rückkehr seit 2010 vermehrt dem Typus return of failure zu entsprechen. Auf der individuellen Ebene von Migranten ist aus der Rückkehr als Handlungsoption (LABRIANIDIS \& KAZAZI 2006), der ja stets entsprechende Freiheitsgrade immanent waren, schneller als erwartet eine Sackgasse mit entsprechenden Zwängen geworden.

Das Grundproblem der Rückkehrmigration ist, neben dem individuellen Entscheidungsdilemma, hauptsächlich in Albanien selbst zu suchen. Hier hat sich an den Rahmenbedingungen nicht nur der Migration, sondern der täglichen Lebenswelt wenig verändert. Allzu sehr sind albanische Politik und Zivilgesellschaft immer noch von Korruption, Klientelpolitik, starren und unzuverlässigen Behördenapparaten sowie von Arbeitslosigkeit, unterqualifizierter Beschäftigung und fehlender Perspektive vor allem für die jüngeren Generationen gekennzeichnet. Das waren genau jene Faktoren, die zahllose Albaner ursprünglich in die Emigration getrieben hatten und welche die albanische Emigration seit zwei Jahrzehnten als Konstante begleiten. Es ist bislang nur bedingt gelungen, im Land selbst eine langfristig tragfähige ökonomische Basis und eine funktionierende Zivilgesellschaft aufzubauen. Insofern ist die Remigration, ebenso wie es die Emigration gewesen war, mit WiLLIAMs \& BALÁsž (2011) stets mit Risiko und Unsicherheit verbunden. Soziale Resilienz ist in Albanien, wie gezeigt wurde, eng mit Elementen von Transnationalismus und Translokalität verbunden. 
Transregionalismus ist ein integraler Bestandteil der albanischen Lebenswelt. „Return [...] constitutes one stage in the migration process" weiß CASSARINO (2004, S. 268) und rückt damit - auch wenn im entsprechenden Schema die problematischen biographischen Komponenten nicht berücksichtigt sind - die Frage des ,level of preparedness“ von Rückkehrern in den Vordergrund (CASSARINO 2004, S. 273).

\section{Transregionalismus als Beitrag zur Rekonzeptualisierung}

In der Zusammenschau der beiden empirischen Befunde respektive des Vergleichs des Unvergleichbaren hinsichtlich der jeweiligen Reaktionen, Resistenzen und Resilienzen offenbaren sich wichtige Gemeinsamkeiten genauso wie elementare Gegensätze. $\mathrm{Zu}$ letzteren zählen der Transformationspfad und die EU-Mitgliedschaft. Hier blickt Lettland auf eine sich stabilisierende Kontinuitätslinie zurück, während Albanien mit mehrfachen Unterbrechungen des von Beginn an schwierigen Weges einer phasenweise krisenhaften Transformation konfrontiert war. Das hatte gravierende Auswirkungen auf die Migrationssysteme: Zwar gab es auch in Lettland zunächst Auswanderungen in relevanten Größenordnungen, allerdings vollzog sich um das Jahr 2000 der Übergang zur Wohlstandsmigration, das heißt zu verstärkten Binnenmigrationen in Form von Suburbanisierungsprozessen speziell in der Hauptstadtagglomeration Riga. Albanien leidet seit über 20 Jahren unter einer massiven Auswanderung. Migration ist hier das allgegenwärtige Muster sozialer Resilienz in Phasen multipler Krisen auf einem insgesamt niedrigen Wohlstandsniveau. Die Veränderungen von Bevölkerungsverteilung und Siedlungssystem dagegen zeigen vergleichbare räumliche Muster. Wachstumsdynamiken verzeichnen vor allem die Hauptstadtregionen und dort insbesondere der peri-urbane Rand.

In Zeiten der Finanz- und Wirtschaftskrise zeigte sich Albanien bislang resistenter. Wegen der Abhängigkeit von Rimessen hängen die krisenhaften Phänomene kausal mit den Problemen in Griechenland zusammen; die exogene Verursachung und die individuelle Betroffenheit dort führen letztlich zu Rückwanderung. In Lettland, wo die Probleme durch das Kreditwesen endogen bedingt sind, führt die Verschuldung nun zu einer neuerlichen Auswanderung. Summa summarum ist in Lettland die neue Auswanderung die Form sozialer Resilienz in Zeiten der Krise; in Albanien ist es die Rückkehr. Ein ähnlicher Input hat unter unterschiedlichen räumlichen Gegebenheiten zu sehr unterschiedlichen Reaktionen geführt.

Die Variabilität der Migrationssysteme wird im gegebenen Zusammenhang von zwei wesentlichen Komponenten bestimmt. Das sind erstens der gesamtgesellschaftliche Transformationspfad und zweitens der soziale Kontext oder der Grad der individuellen Verwundbarkeit der Migranten. Insofern erfordert die theoretische Fassung des Phänomens die Verknüpfung ökonomischer Rationalismen und Determinismen mit den possibilistischen Ansätzen sozialer Netzwerke, Inkorporation und Embeddedness. Monetäre Fragen der Existenzsicherung werden im individuellen Fall mit Belangen von Zugehörigkeit, Identität und Emotionalität verschränkt (vgl. dazu auch LEviTT 2002; Boyd 1989; Salaff 2013, S. 460 und 462). Die gemeinsame Handlungsarena 
solcher Faktoren lässt sich im Transnationalismusdiskurs nicht hinreichend nachzeichnen. Das vorgeschlagene Konzept des Transregionalismus hilft dabei, die räumliche Diversität der Veränderungen von Migrationssystemen in solchen Zusammenhängen besser verstehen zu können.

\section{Literaturverzeichnis}

Albanian Centre for Competitiveness and International Trade (ACIT) (Hrsg.) (2012), Study on the Economic Impact of the Greek Crisis in Albania. Tirana.

Apsite E., Krišsāne Z., Berzins M. (2012), Emigration from Latvia under economic crisis conditions. In: International Proceedings of Economics Development and Research, 31, S. 134-138.

APsite-Berina E. (2013), Starpvalstu migrācija Eiropas Savienībā: Latvijas iedzīvotāju migrācija uz Lielbritāniju [Internationale Migration in der Europäischen Union: Emigration von Lettland in das Vereinigte Königreich]. Riga, Universität Riga, Dissertation.

Arrehag L., SJöberg Ö., S Jöblom M. (2005), Cross-border migration and remittances in a postcommunist society: return flows of money and goods in the Korçë district, Albania. In: South Eastern Europe Journal of Economics, 1, S. 9-40.

BarJaba K., King R. (2005), Introducing and theorising Albanian migration. In: King R., MaI N., Schwandner-Sievers S. (Hrsg.), The new Albanian migration, S. 1-28. Portland (Sussex), Sussex Academic Press.

Basch L., Glick Schiller N., Blanc-Szanton C. (Hrsg.) (1994), Nations Unbound: Transnational Projects, Postcolonial Predicaments, and Deterritorialized Nation-States. London, Routledge.

Betts A. (2011), Multilayered Migration Governance. The Promise of Partnership. In: Kunz R., Lavenex S., Panizzon M. (Hrsg.), The global governance of migration and the role of trans-regionalism (= Routledge advances in international relations and global politics, 89), S. 23-45. New York, Routledge.

Boyd M. (1989), Family and personal networks in international migration: Recent developments and new agendas. In: International Migration Review, 23, 3, S. 638-669.

BRENNER N. (1999), Globalisation as reterritorialisation: the re-scaling of urban governance in the European Union. In: Urban Studies, 36, 3, S. 431-451.

CARLING J. (2012), Collecting, analysing and presenting migration histories. In: VARGAS-SILVA C. (Hrsg.), Handbook of Research Methods in Migration, S. 137-162. Cheltenham Northampton, Edward Elgar Publishing.

Cassarino J.-P. (2004), Theorising Return Migration: The Conceptual Approach to Return Migrants Revisited. In: International Journal of Multicultural Societies, 6, 2, S. 253-279.

Castles St., Miller M. (2009), The Age of Migration. International Population Movements in the Modern World, $4^{\text {th }}$ edition. New York - London, Palgrave Macmillan.

Cerase F. (1974), Expectations and reality: A case study of return migration from the United States to the Southern Italy. In: International Migration Review, 8, S. 245-262.

Dahinden J. (2009), Die transnationale Perspektive. In: terra cognita, 15, S. 16-19.

Deutsche Welle, 8. November 2011

DeZwager N., Gedeshi I., Germenji E., Nikas Ch. (2005), Competing for Remittances. Tirana, International Organization for Migration (IOM). 
DunN K. (2010), Embodied transnationalism: bodies in transnational spaces. In: Population, Space and Place, 16, 1, S. 1-9.

Eglîte P., Markausa I., Pavlina I., Brants M., Brīvprātīgā M. (2006), Prombūtne no Latvijas pēc pievienošanās ES. Darbiem un atpūtai veltītais laiks $2003 \mathrm{~g}$. [Der Weg aus Lettland seit dessen Beitritt zur EU. Zeit für Arbeit und Freizeit im Jahr 2003]. In: Apcerējumi par Latvijas iedzīvotājiem, 12, S. 7-57.

FAIST Th. (2000), The Volume and Dynamics of International Migration and Transnational Social Spaces. Oxford, Oxford University Press.

FAIST Th. (2008), Migrants as transnational development agents: an inquiry into the newest round of the migration-development nexus. In: Population, Space and Place, 14, S. 21-42.

Faist Th. (2013), Transnationalism. In: Gold S.J., NAwYN S.J. (Hrsg.), The Routledge International Handbook of Migration Studies, S. 449-459. London - New York, Routledge.

Fassmann H., Münz R. (2000), Vergangenheit und Zukunft der europäischen Ost-West-Wanderung. In: Fassmann H., Münz R. (Hrsg.), Ost-West-Wanderung in Europa, S. 11-48. Wien et al., Böhlau.

Fielding T. (2010), Migration in a Time of Crisis: A Simple Conceptual Framework Applied to East Asian (= Working Paper, 63). Sussex, Sussex Centre for Migration Research.

Findlay A., Geddes A., McCollum D. (2010), International Migration and Recession. In: Scottish Geographical Journal, 126, S. 299-320.

Foner N. (2000), From Ellis Island to JFK: New York's Two Great Waves of Immigration. New Haven, Yale University Press.

Friedrich K., Schultz A. (Hrsg.) (2008), Brain drain oder brain circulation? Konsequenzen und Perspektiven der Ost-West-Migration (= forum ifl, 8). Leipzig, Leibniz-Institut für Länderkunde.

Gedeshi I., DeZwager N. (2012), Effects of the global crisis on migration and remittances in Albania. In: Sirkeci I., Cohen J.H., Ratha D. (Hrsg.), Migration and Remittances during the Global Financial Crisis and Beyond, S. 237-254. Washington, The World Bank.

Germenji E., Milo L. (2009), Return and labour status at home: evidence from returnees in Albania. In: Southeast European and Black Sea Studies, 9, S. 497-517.

GLICK SCHILLER N. (2003), The centrality of ethnography in the study of transnational migration. In: Foner N. (Hrsg.), American Arrivals: Anthropology Engages the New Immigration, S. 99-128. Santa Fe, School for Advanced Research Press.

Glick Schiller N. (2004), Transnationality. In: Nugent D., Vincent J. (Hrsg.), A Companion to the Anthropology of Politics, S. 44-67. Malden, Blackwell Publishing.

GLICK SCHILlER N. (2005), Transnational Social Fields and Imperialism: Bring a Theory of Power to Transnational Studies. In: Anthropological Theory, 5, 4, S. 439-461.

Glick Schiller N., Basch L.G., Blanc-Szanton C. (1992), Towards a Transnational Perspective on Migration: Race, Class, Ethnicity, and Nationalism Reconsidered. New York, New York Academy of Sciences.

GöLER D. (2005), European Shrinking Regions. Applied Regional Geography in Peripheral Areas (with Case Studies from Albania and Germany) (= Studime Gjeografike, 16). Tirana, Qendra e Studimeve Gjeografike.

GÖLER D. (2007), Entrepreneurship im Transformationskontext - Eine Analyse des regionalen Gründungsgeschehens in Südosteuropa (mit Beispielen aus Albanien und Serbien). In: Europa Regional, 15, S. 23-37.

GöLER D. (2009), Regionale und lokale Auswirkungen von Migration in Albanien. In: Südosteuropa, 56, S. 472-499.

GöLER D. (2011), Returnee's Business in Südosteuropa: Remigration als Entwicklungspotential in Albanien? In: Brunnbauer U., Novinscak K., Voss Chr. (Hrsg.), Gesellschaften in 
Bewegung. Emigration aus und Immigration nach Südosteuropa in Vergangenheit und Gegenwart, S. 205-218. München - Berlin, Otto Sagner.

GöLer D., DoKa Dh. (2013), Should I stay or should I go? Emigration, return-migration and development in Albania - The migration-development-nexus at a dangerous crossroads. In: Pichler R. (Hrsg.), Back to the future? Understanding the turbulences of Albanian transitions from contemporary and historical perspectives. [im Druck]

HaAs H. DE (2010), Migration and Development: A Theoretical Perspective. In: International Migration Review, 44, 1, S. 227-264.

HaUg S. (2008), Migration networks and migration decision-making. In: Journal of Ethnic and Migration Studies, 34, 4, S. 585-605.

Herod A., Wright M.W. (Hrsg.) (2002), Geographies of Power: Placing Scale. Malden, Blackwell Publishing.

Hillmann F., Oswald A.v., Schmelz A. (2012), Editorial on the special issue „Rethinking Migration in Times of Economic Crisis in Europe“. In: Comparative Population Studies - Zeitschrift für Bevölkerungswissenschaft, 37, 1-2, S. 3-8.

HoERder D. (2012), Transnational - transregional - translocal: transcultural. In: VARGAS-SILVA C. (Hrsg.), Handbook of Research Methods in Migration, S. 69-91. Cheltenham - Northampton, Edward Elgar Publishing.

Itzigsohn J., Cabral C.D., Medina E.H., Vazquez O. (1999), Mapping Dominican transnationalism: narrow and broad transnational practices. In: Ethnic and Racial Studies, 22, 2, S. 316-339.

Kilic T., Carletto C., Davis B., Zezza A. (2009), Investing back home. Return migration and business ownership in Albania. In: Economics of Transition, 17, S. 587-623.

KING R. (2002), Towards a New Map of European Migration. In: International Journal of Population Geography, 8, S. 89-106.

King R., Castaldo A., Vullnetari J. (2011b), Gendered Relations and Filial Duties Along the Greek-Albanian Remittance Corridor. In: Economic Geography, 87, S. 393-419.

King R., Skeldon R. (2010), 'Mind the Gap!' Integrating Approaches to Internal and International Migration. In: Journal of Ethnic and Migration Studies, 36, 10, S. 1619-1646.

King R., Skeldon R., Vullnetari J. (2008), Internal and International Migration: Bridging the Theoretical Divide (= Working Paper, 52). Sussex, Sussex Center for Migration Research.

King R., Uruçı E., Vullnetari J. (2011a), Albanian migration and its effects in comparative perspective. In: Journal of Balkan and Near Eastern Studies, 13, 3, S. 271-286.

Kivisto P. (2001), Theorizing transnational immigration: a critical review of current efforts. In: Ethnic and Racial Studies, 24, 4, S. 549-577.

Krišjāne Z., Eglīte P., Bauls A., Lulle A., Bērzinš M., Brants M., Cunska Z., Gnedoviska I., Ivbulis B., Krūzmētra Ž., Küle L., Markavsa I.M., Niklass M., Pavlina I., Titova N., VAnaga S., Vilcinš A., Zarina I.B. (2007), Darbaspēka ǵeogrāfiskā mobilitāte [Die räumliche Mobilität von Arbeitskräften]. Rīga, Latvijas Universitāte.

KRIŠJĀNe Z. (2008), Labour Migration Processes after European Union Accession in Latvia. In: Friedrich K., Schultz A. (Hrsg.), Brain drain oder brain circulation? Konsequenzen und Perspektiven der Ost-West-Migration (= forum ifl, 8), S. 63-71. Leipzig, LeibnizInstitut für Länderkunde.

KRIŠJĀne Z., Berzins M., Bauls A. (2009), New Labor Migration Trends: Evidence from Latvia. In: Geographical Review of Japan, Series B 81, 1, S. 34-46.

Labrianidis L., HatziproKopiou P. (2005), The Albanian migration cycle: migrants tend to return to their country of origin after all. In: King R., Mai N., Schwandner-Sievers St. (Hrsg.), The new Albanian migration, S. 93-117. Portland (Sussex), Sussex Academic Press. 
LABRIANIDIS L., KAZAZI B. (2006), Albanian return-migrants from Greece and Italy: Their impact upon spatial disparities within Albania. In: European Urban and Regional Studies, 13, S. 59-74.

Levitt P. (1999), Social Remittances: A Local Level, Migration Driven Form of Cultural Diffusion. In: International Migration Review, 32, 124, S. 926-949.

Levitt P. (2001a), The Transnational Villagers. Berkeley - Los Angeles, University of California Press.

LevitT P. (2001b), Transnational migration: Taking stock and future directions. In: Global Networks, 1, 3, S. 195-216.

LevitT P. (2002), The ties that change: Relations to the ancestral home over the life cycle. In: Levitt P., Waters M. (Hrsg.), The Changing Face of Home: The Transnational Lives of the Second Generation, S. 123-144. New York, Russell Sage Foundation.

Levitt P., De Wind J., Vertovec S. (2003), International perspectives on transnational migration: an introduction. In: International Migration Review, 37, S. 565-375.

Levitt P., Glick Schiller N. (2004), Transnational Perspectives on Migration: Conceptualizing Simultaneity. In: International Migration Review, 38, S. 1002-1039.

Levitt P., Jarowska N. (2007), Transnational Migration Studies: Past Developments and Future Trends. In: Annual Review of Sociology, 33, S. 129-156.

Lichtenberger E. (1984), Gastarbeiter. Leben in zwei Gesellschaften. Wien et al., Böhlau.

Marston S.A., Hohn P.J., Woodward K. (2005), Human geography without scale. In: Transactions of the Institute of British Geographers, 30, 4, S. 416-432.

Müller D., Munroe D. (2008), Changing rural landscapes in Albania: Cropland abandonment and forest clearing in the postsocialist transition. In: Annals of the Association of American Geographers, 98, S. 855-876.

Müller-Mahn D. (2011), Die Auflösung von Norden und Süden: neue Raumbilder als Herausforderungen für die Geographische Entwicklungsforschung. In: Gebhardt H., Glaser R., RadtKe U., Reuber P. (Hrsg.), Geographie. Physische Geographie und Humangeographie, 2. Auflage, S. 763-775. Heidelberg, Spektrum Akademischer Verlag.

NIKAS Ch., KING R. (2005), Economic growth through remittances: Lessons from the Greek experience of the 1960s applicable to the Albanian case. In: Journal of Southern Europe and the Balkans, 7, S. 235-257.

PAPE U. (2012), Welt: Rücküberweisungen von Migranten steigen. In: Migration und Bevölkerung Newsletter, 10, S. 7-8.

Pickles J. (2010), The spirit of post-socialism: Common spaces and the production of diversity. In: European Urban and Regional Studies, 17, 2, S. 127-140.

Pickles J., Smith A. (Hrsg.) (1998), Theorising Transition: The Political Economy of Postcommunist Transformations. London, Routledge.

Portes A. (2001), Introduction: the debates and significance of immigrant transnationalism. In: Global Networks, 1, 3, S. 181-194.

Portes A. (2003), Conclusion: Theoretical convergencies and empirical evidence in the study of immigrant transnationalism. In: International Migration Review, 37, 3, S. 874-892.

Portes A., Guarnizo L.E., Landolt P. (1999), The study of transnationalism: pitfalls and promise of an emergent research field. In: Ethnic and Racial Studies, 22, 2, S. 217-237.

Pries L. (2001), New transnational social spaces. International migration and transnational companies in the early twenty-first century. London, Routledge.

PrIES L. (2005), Configurations of geographic and societal spaces: a sociological proposal between ,methodological nationalism' and the ,spaces of flows'. In: Global Networks, 5, S. 167-190. 
Pries L. (Hrsg.) (2008), Rethinking Transnationalism. The Meso-link of Organisations. London, Routledge.

RIESter A. (2012), Resilience of Remittances during the Global Financial Crisis and the Entrenchment of Migration. In: Sirkeci I., Cohen J.H., Ratha D. (Hrsg.), Migration and Remittances during the Global Financial Crisis and Beyond, S. 141-147. Washington, The World Bank.

Salaff J.W. (2013), Return Migration. In: Gold S.J., NawYN S.J. (Hrsg.), The Routledge International Handbook of Migration Studies, S. 460-468. London - New York, Routledge.

Sмiтн M.P. (2005), Transnational urbanism revisited. In: Journal of Ethnic and Migration Studies, 31, 2, S. 235-244.

Sмith A., Timár J. (2010), Uneven transformations: Space, economy and society 20 years after the collapse of state socialism. In: European Urban and Regional Studies, 17, 2, S. 115-125.

Smith M.P., Guarnizo L.E. (Hrsg.) (1998), Transnationalism from Below. New Brunswick New Jersey, Transaction Publishers.

United Nations Development Programme (UNDP) (Hrsg.) (2000), Albanian Human Development Report 2000. Tirana, UNDP.

Velayutham S., Wise A. (2005), Moral economies of a translocal village: obligation and shame among South Indian transnational migrants. In: Global Networks, 5, 1, S. $27-47$.

Vertovec S. (2001), Transnationalism and identity. In: Journal of Ethnic and Migration Studies, 27,4, S. 573-582.

Vertovec S. (2004a), Cheap calls: The social glue of migrant transnationalism. In: Global Networks, 4, 2, S. 219-224.

Vertovec S. (2009), Transnationalism. London - New York, Routledge.

VoICE OF AMERICA/GIIROKASTËr, 15. Februar 2012

Vullnetari J. (2007), Albanian Migration and Development: State of the Art Review (= IMISCOE Working Paper, 18). Sussex, Sussex Centre for Migration Research.

Vullnetari J. (2012), Albania on the move. Links between internal and international migration (= IMISCOE Research). Amsterdam, Amsterdam University Press.

Vullnetari J., King R. (2008), Does your granny eat grass? On mass migration, care drain and the fate of older people in rural Albania. In: Global Networks, 8, S. 139-171.

Waldinger R., FitzGerald D. (2004), Transnationalism in question. In: American Journal of Sociology, 109, S. 1177-1195.

Williams A., Balásž V. (2011), Migration, Risk, and Uncertainty: Theoretical Perspectives. In: Population, Space and Place, 18, S. 197-180.

World B ANK (Hrsg.) (2011), Migration and Remittances Factbook 2011. 2. Auflage. Washington, World Bank.

Webseiten:

Click H+K Research (2012), Socialo tiklu lietojums Latvija [Die Nutzung sozialer Netzwerke in Lettland]. - http://twitpic.com/a6pkb6/full (zuletzt aufgerufen am 16.8.2013)

Global FinanCe 2013. - http://www.gfmag.com/gdp-data-country-reports.html\#axzz2Yd6Powmm (zuletzt aufgerufen am 29.7.2013)

United Nations Population Division (2013), International Migration Policies 2013. - http:// www.un.org/en/development/desa/population/ (zuletzt aufgerufen am 29.7.2013)

WoRLd B ANK (2013), Migration and Remittances. - http://www.worldbank.org/migration (zuletzt aufgerufen am 7.8.2013) 